\title{
Griseldaonline. Una sfida al labirinto letterario
}

\section{Nicola Bonazzi \& Andrea Campana, Università di Bologna}

\begin{abstract}
This article presents the Italian literary magazine Griseldaonline that was created in 2002 by a group of young scholars from the University of Bologna. The magazine has encouraged the production of new forms of critical literary writing conceived specifically for the web.
\end{abstract}

II magazine letterario Griseldaonline (www.griseldaonline.it), trae il suo nome dalla protagonista dell'ultima novella del Decameron di Boccaccio, ovvero da un personaggio complesso, contraddittorio, che lascia spiazzati gli esegeti per le sue ambivalenze (che arrivano a parere aporie), tra magnanimità e sottomissione, forza morale e pusillanimità o ignavia: proprio per quest'aura d'enigma, il personaggio boccacciano in questione è stato scelto dai fondatori della rivista quale vessillo critico di dialogo senza pregiudizi, sempre sul filo dell'azzardo, della novità, anche anti-accademica, con ciò che è contraddittorio per definizione allo sguardo interpretativo, la letteratura appunto. Griseldaonline è dunque un portale dove tutti possono avere accesso al dibattito su letteratura e critica letteraria, siano essi docenti, scrittori, poeti, soggetti provenienti dal mondo scolastico e universitario o, più in generale, da quello delle professioni. Neppure il problema letterario, seppure resti prevalente, viene trattato sul sito in maniera univoca ed esclusiva: anche altre aree espressive sono ammesse alla riflessione (di volta in volta, il cinema, l'antropologia, la sociologia, la storia delle idee).

II sito - nato ufficialmente nel 2002 - riceve i finanziamenti dalla Fondazione Cassa di Risparmio in Bologna ed è stato fin dalle origini attivamente sostenuto da Gian Mario Anselmi, ordinario di Letteratura italiana al Dipartimento di Filologia Classica e Italianistica dell'Alma Mater Studiorum (gli altri co-fondatori, insieme a lui, sono: Nicola Bonazzi, Elisabetta Menetti, Francesca Tomasi, Claudio Tubertini e Carlo Varotti); esso, nel corso degli anni, ha assunto sempre più l'aspetto di un luogo sperimentale, capace di ospitare una forma nuova di saggistica, che tenga assieme divulgazione e dottrina, incisività esegetica e comunicazione. Una delle peculiarità di questa nuova 'forma', che si estrinseca sulle pagine web di Griseldaonline, consiste nel formato dei contributi pubblicati, digitali, disponibili in versione stampabile, costellati di links testuali e iconografici. 
Insomma, nulla di simile allo stile del saggio scientifico tradizionale. Come scrive Elisabetta Menetti nell'introduzione alla raccolta in formato cartaceo di alcuni saggi della rivista, "l'esperimento di Griseldaonline riguarda una nuova forma saggistica, che sia capace di tenere insieme profondità ermeneutica e leggerezza compositiva e che sia in grado di immaginare un percorso interpretativo al contempo lineare e a rete" (Menetti 2).

Si tratta di una scelta metodologica di partenza quasi inevitabile, suggerita dalla nuova forma di testualità che il medium elettronico implica. Leggerezza e complessità, come aveva ben presentito il Calvino delle Lezioni americane, sono le caratteristiche del nuovo mezzo di comunicazione, che nelle sue forme più compiute asseconda l'accessibilità a se stesso riconfigurando materialmente il testo. Naturalmente si tratta di una riconfigurazione che ha poi ampie ricadute sul piano ermeneutico. Così, se da un lato l'esperienza dell'occhio alle prese con il nuovo formato elettronico esige il confronto con testi ampiamente paragrafati e corredati possibilmente di un apparato iconografico che ne spezzi la coriacea continuità, dall'altro l'articolazione verticale del testo elettronico, in via teorica illimitata, attraverso connessioni e finestre di rete (links), che permettono all'utente di muoversi con libertà tra elementi diversi $e$ in uno spazio perennemente mutevole, instaura un rapporto privilegiato con un nuovo tipo di lettore (un lettore dunque attivo), in grado di creare da sé i percorsi ermeneutici all'interno del mezzo, sulla base delle proprie esigenze o delle competenze già acquisite.

D'altro canto, come teorizza George Landow, uno dei principali studiosi dell'ipertesto, quando i lettori procedono attraverso una rete di testi, spostano continuamente il centro della loro indagine: il punto di focalizzazione provvisorio dell'ipertesto cambia in continuazione proprio grazie alla partecipazione attiva del lettore: è tale lettore che trasforma i propri interessi nel principio organizzativo dell'indagine, così da fare dell'ipertesto un sistema infinitamente decentrabile e ricentrabile (Landow 79).

Naturalmente Griseldaonline, nata molti anni dopo che le prime riflessioni teoriche sul nuovo medium erano state esperite, di tali riflessioni si è giovata, inizialmente in via del tutto empirica (ed è inevitabile per chi arrivava da esperienze puramente letterarie), ma focalizzando poi lungo il percorso l'inesauribile ricchezza interpretativa del formato elettronico. Intertestualità, polifonia, apertura testuale, decentramento: tutti concetti utili a definire gli statuti fluidi e liberi del nuovo mezzo.

Una prima sezione in cui si articola il portale contiene gli interventi critici sui temi che via via la redazione propone agli studiosi, come in una sorta di call for papers allargato alla comunità virtuale della rete. Basta leggere i titoli dei vari percorsi tematici, oggi arrivati al numero di 11, per rendersi conto della versatilità e multidisciplinarità della ricerca messa in campo: Inferni, L'altro, // 
corpo, I/ nemico, Ai giovani, Rifiuti scarti esuberi, A rovescio, Metamorfosi, Verità $e$ immaginazione, fino ai più vicini Ecologia dello sguardo e Denaro. Lo scopo di partenza di ciascun percorso è di offrire un'analisi del tema in oggetto nella letteratura non solo italiana, ma anche internazionale; a interventi di taglio puramente letterario, tuttavia, lo si è già detto, si vanno ad affiancare, spesso, interventi più teorici (perciò più generali), di contorno e inquadramento. Nella sezione "Sonde" si incontrano nomi noti (Ermanno Cavazzoni, Gianni Celati, Valerio Magrelli, Edoardo Sanguineti, solo per citarne alcuni), talvolta contributori con testi scritti in prima persona, altre volte semplicemente "intervistati" a cura della redazione. Percorrendo in profondità il portale si possono incontrare altre sezioni non più in evidenza, come "Formazione e didattica," realizzata in collaborazione con ADI-Scuola (Associazione degli Italianisti Italiani), riservata a testi più adatti ad un pubblico scolare, o comunque organizzati secondo un criterio di maggiore limpidezza di linguaggio; o, ancora, la sezione "Informatica" che tentava di aggiornare sulle novità dell'informatica umanistica, fornendo strumenti essenziali per accostare questa disciplina decisiva nella nostra epoca, compreso un Manuale di informatica umanistica redatto da Francesca Tomasi, scaricabile quale open source. Non vanno infine sottovalutate le Risorse online promosse dal portale, che includono l'indicazione dei principali siti web esistenti in fatto di Didattica, Monografie ipertestuali, Archivi testuali, Informatica umanistica.

Di recente, il sito si è arricchito di una sua storia letteraria, anch'essa gratuita, la Letteratura italiana di Griselda, condotta da 24 studiosi, giovani o già affermati, seguendo criteri non convenzionali: ogni capitolo ha infatti di mira l'indagine di una porzione di storia letteraria italiana da una prospettiva eminentemente 'europea', intesa a mettere in evidenza le relazioni tra i nostri autori e quelli stranieri; la trattazione, inoltre, può in ogni momento giovarsi di links extratestuali impensabili per la manualistica cartacea, rimandando spesse volte a immagini, testi elettronici, mappe, ecc.

II tentativo è di realizzare una storia letteraria animata e leggibile (per questo un'altra prospettiva privilegiata è quella biografica), in grado di superare specialismi e arroccamenti accademici, destinati spesso a inaridire una materia che invece, per sua stessa natura, dovrebbe aprire orizzonti e percorsi di pensiero. Indicative e pienamente esplicative le parole del fautore principale dell'impresa, Gian Mario Anselmi, nella Presentazione del progetto:

Nella novità del mezzo vogliamo infatti riscoprire la dignità dell'esercizio critico come etica della lettura forte e pienamente responsabile: ovvero varietà consapevole dell'interpretazione nell'unità e coesione dei fini. E' un lavoro che si fa e si farà nel tempo, non sarà mai davvero compiuto e potrà presupporre magari in futuro, ad esempio, anche più capitoli di più autori dedicati allo stesso protagonista o allo stesso periodo, ognuno con 
la sua peculiare chiave di lettura: ma diversamente che nella vecchia pratica decostruzionista il radicamento nella storia, nel contesto e nei diritti inalienabili del testo nella sua 'verità' filologica sarà essenziale e il lettore sceglierà cosi con piena consapevolezza storica la lettura a lui più congeniale $o$ le terrà presenti tutte per avere il senso vivo della dialogicità tra grandi classici e loro attuali interpreti. Interpreti preferibilmente giovani, non logorati da troppe, decennali milizie e malizie accademiche. Questa vorrebbe anche presentarsi infatti come la storia letteraria di nuove generazioni di studiosi che hanno aderito con entusiasmo all'impresa, cui chi scrive, e sulla scorta di lezioni di grandi e ormai classici maestri, ha offerto solo la sponda del coordinamento, dell'esperienza e della passione non sopita per la saggezza antica della letteratura. (Anselmi)

È già in cantiere una versione a stampa, più succinta, di questa plurivoca Letteratura, che, scritta nello stile più colloquiale e leggero, niente affatto paludato o professorale, adatto alla fruizione in rete, stabilirà con quella online lungi dal sostituirsi ad essa - uno scambio biunivoco, osmotico, di apporti e rimandi, ciò che costituirà un fatto sostanzialmente innovativo nel panorama culturale nostrano.

Per concludere, qualche dato su Griseldaonline: dopo che per molti anni il progetto grafico il layout, la gestione e l'aggiornamento del periodico sono stati curati da Claudio Tubertini di ArchetipoLibri di Bologna, ora la cura è affidata all'azienda Mediavision di Bologna. II direttore responsabile è Gian Mario Anselmi (Università di Bologna); la direttrice di redazione è Elisabetta Menetti (Università di Modena e Reggio Emilia); mentre coordinatore redazionale è Nicola Bonazzi. II Comitato scientifico è così composto: Gian Mario Anselmi, Nicola Bonazzi, Andrea Campana, Francesco Citti, Magda Indiveri, Nicolò Maldina, Elisabetta Menetti, Lucia Pasetti, Andrea Severi.

Per contattare la rivista: info@griseldaonline.it 


\section{Works Cited}

Anselmi Gian Mario. Presentazione a Storia della letteratura italiana di Griseldaonline

(http://www.letteraturaitalianaonline.com/Storia\%20della\%20letteratura\%20italiana.

Data ultima visione: 20/01/2012)

Landow G. L'ipertesto. Tecnologie digitali e critica letteraria, Milano, Bruno Mondadori Editore, 1998. Print.

Menetti E. "Griseldaonline e le forme della comunicazione letteraria." AA.VV.

Griseldaonline. Una rivista letteraria nell'era digitale, Bologna: Archetipolibri, 2008. Print. 